\title{
Sumoylation Inhibitor TAK-981
}

National Cancer Institute

\section{Source}

National Cancer Institute. Sumoylation Inhibitor TAK-981. NCI Thesaurus. Code C156270.

A small molecule inhibitor of sumoylation, with potential immune-activating and antineoplastic activities. Upon intravenous administration, TAK-981 targets and covalently binds to the small ubiquitin-like modifier (SUMO; small ubiquitin-related modifier) protein, forming an adduct with SUMO protein (TAK-981-SUMO adduct). This prevents the transfer of SUMO from the SUMO-activating enzyme (SAE) to SUMOconjug ating enzyme UBC9. This prevents SUMO conjug ation to lysine residues on target proteins and abrogates many sumoylated protein-mediated cellular processes that play key roles in tumor cells, including proliferation, DNA repair, metastasis and survival. In addition, by preventing sumoylation, TAK-981 is able to increase the production of type 1 interferon (IFN), thereby increasing type 1 IFN-mediated signaling, activating innate effector cells and enhancing the antitumor innate immune responses. This may further increase tumor cell killing. Sumoylation, a post-translational modification that attaches the SUMO protein to target proteins, plays a key role in regulating their activity, function, subcellular localization and stability. Sumoylation also plays a key role in inhibiting innate immune responses, specifically by inhibiting the pattern recognition receptor (PRR) pathway and preventing type 1 IFN expression. Abnormal sumoylation of target proteins is associated with many cancers. 